\title{
Coupling Effect of Strain Rate and Freeze-Thaw Temperature on Dynamic Mechanical Properties and Fractal Characteristic of Saturated Yellow Sandstone
}

\author{
Peng Wu $\mathbb{D}^{1,2}$ Lianying Zhang $\mathbb{D}^{1},{ }^{1}$ Xianbiao Mao, ${ }^{2}$ Yanlong Chen $\mathbb{D}^{2},{ }^{2}$ Ming $\mathrm{Li}^{2}{ }^{2}$ Liang Chen, ${ }^{3}$ \\ and Lan Wang ${ }^{4}$ \\ ${ }^{1}$ Civil Engineering Department, Xuzhou Institute of Technology, Xuzhou, China 221000 \\ ${ }^{2}$ State Key Laboratory for Geomechanics and Deep Underground Engineering, China University of Mining \&Technology, Xuzhou, \\ China 221116 \\ ${ }^{3}$ State Key Laboratory of Coal Resources and Safe Mining, China University of Mining and Technology, Xuzhou 221116, China \\ ${ }^{4}$ School of Mechanics and Civil Engineering, China University of Mining and Technology, Xuzhou 221116, China
}

Correspondence should be addressed to Lianying Zhang; zhanglianying@126.com and Yanlong Chen; chenyanlong@cumt.edu.cn

Received 22 June 2021; Accepted 31 August 2021; Published 26 September 2021

Academic Editor: Yu Wang

Copyright (c) 2021 Peng Wu et al. This is an open access article distributed under the Creative Commons Attribution License, which permits unrestricted use, distribution, and reproduction in any medium, provided the original work is properly cited.

\begin{abstract}
Strain rate is not only an important influence factor for deformation property but also an important parameter for analyzing the dynamic mechanical behavior of rock material. In this study, the dynamic compressive mechanical properties of saturated yellow sandstone at four strain rates and six freeze-thaw temperatures are investigated by using the SHPB test system. The coupling effect of strain rate and freeze-thaw temperatures on the mechanical parameters of rock material are discussed in detail, and the relationship formula of peak strain and dissipated energy with strain rate and freeze-thaw temperature are also established. Finally, the fractal dimension characteristic of fracture specimens with the strain rate and temperature are analyzed by using the fractal dimension method. The research results indicate that (1) with the increase of strain rate, the increase speed of peak strain, peak strength, and dissipated energy at medium strain rate level was obviously higher than that at high strain rate level, indicating that the strain rate effect weakened at high strain rate. (2) Freeze-thaw temperature can improve the brittlenessductile transformation rate of saturated specimens. (3) According to the strain rate sensitivity coefficient, at room temperature, the strain rate effects on peak strain and peak strength are weakest, while at $-20^{\circ} \mathrm{C} \sim-30^{\circ} \mathrm{C}$, they are most significant. In addition, the strain rate effect on dissipated energy is significant at room temperature, while weakest at $-30^{\circ} \mathrm{C}$. (4) The fractal dimension gradually increases with strain rate increasing or freeze-thaw temperature decreasing, indicating that the freeze-thaw environment has a positive function for increasing the damage and fracture degree of specimens for saturated specimen. Our research results can provide an extremely important theoretical basis for the dynamic disaster prevention and structural design of rock engineering in cold regions.
\end{abstract}

\section{Introduction}

The dynamic mechanical properties of rock material are an extremely important evaluation index for rock structural engineering stability, such as tunnel excavation, blasting engineering, mining excavation, and oil drilling engineering. Therefore, the structural design and stability assessment of underground engineering need to take the dynamic mechanical properties of rock material into account for preventing the impact of earthquake and impact load on the structure strength $[1,2]$. Strain rate is an extremely effective parameter which can reflect the deformation and dynamic mechanical behaviors of rock materials. Meanwhile, the mechanical properties of rock material are closely related to the strain rate [3-5]. According to the amplitude, it can be divided into the low strain rate $\left(10^{-5}-10^{-2} \mathrm{~s}^{-1}\right)$, medium strain rate $\left(10^{-2}-10^{2} \mathrm{~s}^{-1}\right)$, high strain rate $\left(10^{2}-10^{4} \mathrm{~s}^{-1}\right)$, and ultrahigh strain rate (more than $10^{4} \mathrm{~s}^{-1}$ ) $[2,6-8]$. In the lower strain rate range, the material strength 
parameter gradually increases with the increase of strain rate, while rapidly increases under the medium strain rate condition. At the high or ultrahigh strain rate level, the strain rate effect of the material strength parameter becomes weak again [9]. The split Hopkinson pressure bar (SHPB) system, as the most commonly used and stable dynamic test device, has been widely used to obtain the dynamic mechanical properties of rock or rock-like materials at the high strain rate. In fact, the strain rate effect of various rock-like materials, e.g., concrete, earth-rock aggregate, and rock, have been investigated and achieved fruitful research results by experimental and numerical simulation methods [10-17]. However, the dynamic mechanical properties of rock materials are not only related to the strain rate but also closely related to the freeze-thaw (F-T) effect. The coupling influence of temperature and impact loading was ignored in the above investigation.

In fact, many rock engineering techniques in cold regions, such as highway construction, open-pit slope stability, and roadway and tunnel excavation, are concerned with the freeze-thaw problem of rock materials. Hence, the design of these engineering must fully take the impact of freezethaw effect on rock mechanical properties into account. At present, a lot of studies have been carried out on the freeze-thaw effect of rock materials under static or dynamic loading conditions [18-25]. However, the above works were mainly limited to static or dynamic mechanical investigation by considering the effect of freeze-thaw cycle times at different strain rates or freeze-thaw temperatures at low or medium strain rate. This does not reveal the influence of freeze-thaw temperature on the dynamic impact mechanical properties of rock materials subjected to the high strain rate loading condition.

In addition, the fragmentation distribution characteristic of specimens after impact loading are also an extremely important index for evaluating the damage degree of rock materials by statistical or fractal methods. A large number of investigations related to the failure characteristics of rock materials have been carried out at different strain rates, stress state, high temperatures, or freeze-thaw effect [9, 26-28]. However, few reports about the coupling effect of the medium-high stain rate and freeze-thaw temperature on dynamic fracture characteristics have been published in literature. In this paper, we mainly focus on the following two issues for detailed discussion:

(1) How does the strain rate affect the dynamic mechanical properties of rock materials subjected to different freeze-thaw temperatures and whether the strain rate hardening effect still exists?

(2) How do the strain rate and freeze-thaw temperature affect the fracture degree of specimens?

Based on the above proposed problems, the dynamic compressive test of saturated yellow sandstone is carried out under different strain rates and temperatures. Then, the coupling effect of strain rate and freeze-thaw temperatures on the dynamic mechanical behaviour of rocks are analyzed in detail and the expression formula of dynamic mechanical parameters related to temperature and strain rate are also deduced in Section 3. Finally, after the impact test, we collected the fragments of saturated yellow sandstone and analyzed its fractal characteristic by using the fractal dimension calculation method. Our research results can provide an extremely important theoretical basis for the dynamic disaster prevention and structural design of rock engineering in cold regions.

\section{Experimental}

2.1. Material Preparation. In this study, the test rock material was yellow sandstone, gathered from the Haerwusu open-pit coal mine (Figure 1) located in Inner Mongolia Autonomous Region, China. The main mineral contents were $54 \%$ albite, $19.9 \%$ quartz, $17.4 \%$ anorthoclase, $5.8 \%$ laumontite, and $2.9 \%$ santafeite. The sandstone specimens were taken from the same block and cut into cylinders $50 \mathrm{~mm}$ in diameter and $25 \mathrm{~mm}$ in height with errors less than $0.05 \mathrm{~mm}$ in height and $0.02 \mathrm{~mm}$ in diameter [9]. Then, the nondestructive ultrasonic $\mathrm{P}$-wave velocity tests carried out in specimen indicated that the yellow sandstone materials exhibit an obvious isotropic behavior. Its $\mathrm{P}$-wave and density were, respectively, $1.58 \mathrm{~km} / \mathrm{s}$ and $2287 \mathrm{~kg} / \mathrm{m}^{3}$ with the average uniaxial compressive strength of $50.89 \mathrm{MPa}$, elastic modulus 5.67 GPa, and Poisson's ratio 0.22.

2.2. Testing Equipment. The main testing equipment included the freeze-thaw testing system, SHPB testing system, and particle size screening device for separating fragment. Firstly, the JC-ZDR-5 freeze-thaw testing machine was used to treat the saturated yellow sandstone materials with eight freeze-thaw cycles under normal temperature $\left(25^{\circ} \mathrm{C}\right)$ and low temperatures $\left(-5^{\circ} \mathrm{C},-10^{\circ} \mathrm{C},-15^{\circ} \mathrm{C},-20^{\circ} \mathrm{C}\right.$, and $\left.-30^{\circ} \mathrm{C}\right)$. Then, an impact loading test was carried out on a $50 \mathrm{~mm}$ diameter SHPB test system, which consists of the loading drive system, pressure bar system, energy absorption system, and signal acquisition system, as shown in Figure 2. During the impact test, the striker bar could obtain different impact speeds by adjusting the air pressure produced by high-pressure nitrogen tank, and then, the striker bar collided with the incident bar with the occurrence of incident plus or stress plus in the incident bar which would be transmitted to the free end of the rock specimen. Some incident pulses were returned to the incident bar as reflected pulse, while others were transmitted to the transmission bar as a transmitted pulse. All of the stress plus information in the two bars would be recorded into a signal acquisition system by the strain gauge on the elastic compression bar. In this study, the elastic modulus and wave velocity of incident bar and transmission bar are, respectively, 210GPa and $5450 \mathrm{~m} / \mathrm{s}$ with the length $25 \mathrm{~mm}$ and diameter $50 \mathrm{~mm}$ of incident bar. After the impact test, the particle size screening device was used to research the fractal characteristic of the fragment by using the fractal dimension method.

2.3. Testing Procedure. In this study, the specific test procedures (Figure 2) were as follows: 


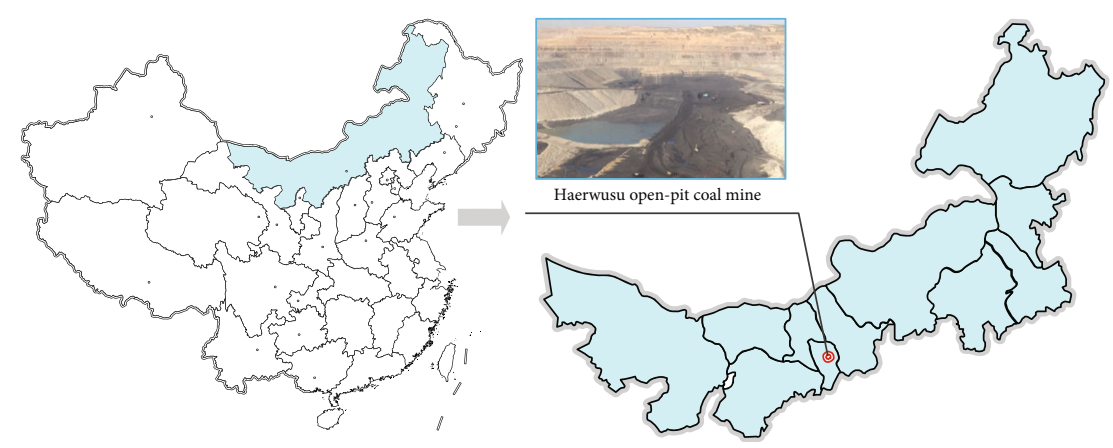

Figure 1: Sampling location.

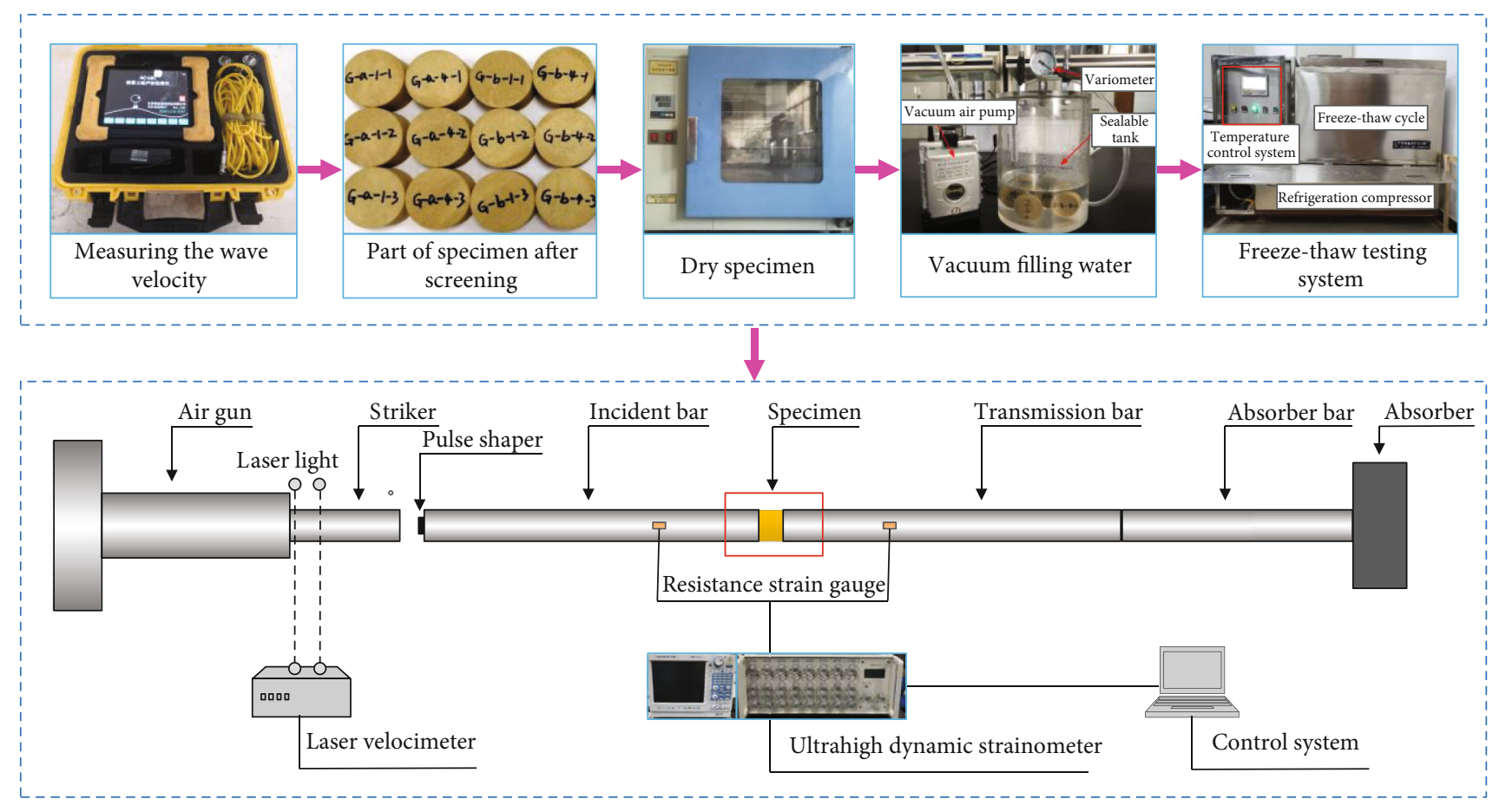

Figure 2: Structure characteristic of the SHPB and testing procedure.

(1) The saturated experimental procedures: the P-wave velocity of nature specimen was firstly monitored. The specimens with the same $\mathrm{P}$-wave velocity (approximately $1580 \mathrm{~m} / \mathrm{s}$ ) were selected to be dried in an incubator for 24 hours at $105^{\circ} \mathrm{C}$. Next, the selected specimens were placed in the vacuum pumping saturation system (VPSS) to extract gas with water injection for 12 hours until the bubblefree overflow from the surface of the specimen. Then, the air pressure was adjusted in the sealed tank to the atmospheric pressure and kept for 12 hours. When the quality change of the specimen was less than $0.01 \mathrm{~g}$ per hour, it can be considered that it has reached the water saturation state

(2) The freeze-thaw cycle test procedures: firstly, the saturated yellow sandstone specimens were taken out from the sealed tank. In order to eliminate the effect of moisture content, the saturated yellow sandstone specimens with the same moisture content were selected as the test specimens and divided into 24 groups with five specimens of each group. The average saturated moisture content was about 5.71\%. Then, the saturated specimens were placed in the freeze-thaw cycle tank and frozen to different temperatures $\left(25^{\circ} \mathrm{C},-5^{\circ} \mathrm{C},-10^{\circ} \mathrm{C},-15^{\circ} \mathrm{C},-20^{\circ} \mathrm{C}\right.$, and $-30^{\circ} \mathrm{C}$ ) with eight freeze-thaw cycles. In addition, the temperature-fall stage, freezing stage, temperature-rise stage, and thawing stage lasted for $2 \mathrm{~h}, 6 \mathrm{~h}, 1 \mathrm{~h}$, and $6 \mathrm{~h}$, respectively; the total time of one freeze-thaw cycle is $15 \mathrm{~h}$. The temperature history curve of the freeze-thaw experiments is displayed in Figure 3

(3) The impact load test procedures: the specimen was placed between the incident bar and transmission bar; the two ends of which were coated with the vaseline to reduce the friction effect at the specimen- 


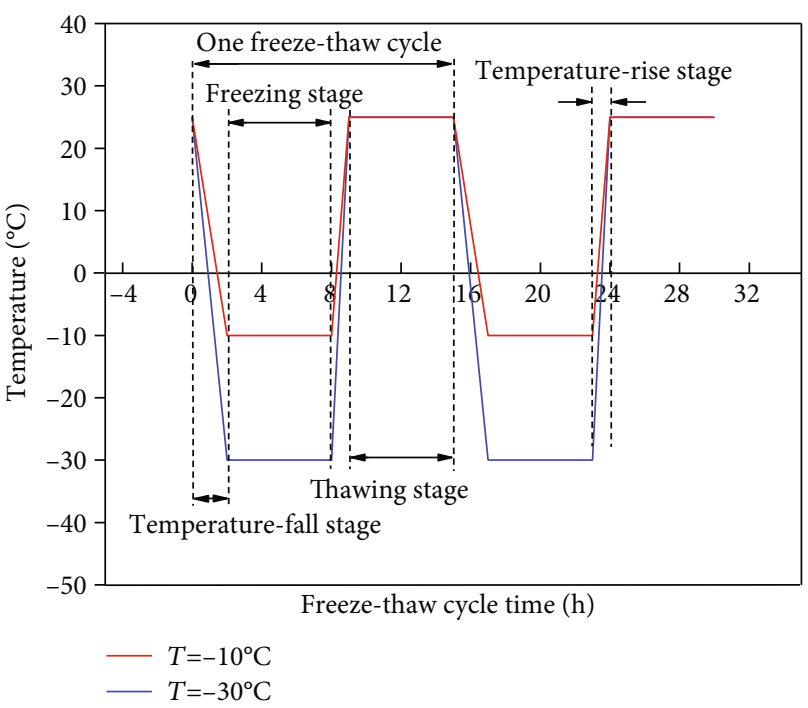

FIGURE 3: Temperature prole of the yellow sandstone specimens undergoing the F-T cycle.

incident bar or specimen-transmission bar interface. Then, an impact gas pressure $(0.2 \sim 0.35 \mathrm{MPa})$ were, respectively, applied to the saturated specimens at the same temperatures to produce different strain rates. The average strain rate ranges of saturated yellow sandstone in this study were from $74.22 \mathrm{~s}^{-1}$ to $169.41 \mathrm{~s}^{-1}$. In this experiment, there were 6 groups of temperature and 4 groups of strain rate. A total of 24 groups of experiments were combined in pairs, and each experiment was repeated 5 times

(4) After the impact test, the fragments of specimens were collected and divided into different particle size ranges by using a classifying screen. Then, the fragment mass in different particle size ranges was measured, and the fractal characteristics were analyzed to reflect the coupling effect of freeze-thaw temperature and strain rate on the damage degree of saturated yellow sandstone

\subsection{Data Processing}

2.4.1. Dissipated Energy Calculation Process. Based on the one-dimensional stress wave theory and the uniform stress assumption, the stress $\sigma(t)$, strain $\varepsilon(t)$, and strain rate $\dot{\varepsilon}(t)$ of each specimen can be calculated by using three-wave analysis methods $[2,24]$.

$$
\left\{\begin{array}{l}
\dot{\varepsilon}=\frac{C_{s}}{l_{s}}\left[\varepsilon_{i}(t)-\varepsilon_{r}(t)-\varepsilon_{t}(t)\right], \\
\varepsilon=\frac{C_{s}}{l_{s}} \int_{0}^{t}\left[\varepsilon_{i}(t)-\varepsilon_{r}(t)-\varepsilon_{t}(t)\right] d t, \\
\sigma=\frac{A_{0} E_{0}}{2 A_{s}}\left[\varepsilon_{i}(t)+\varepsilon_{r}(t)+\varepsilon_{t}(t)\right],
\end{array}\right.
$$

where $\varepsilon_{i}(t), \varepsilon_{r}(t)$, and $\varepsilon_{t}(t)$, respectively, represent the strain signals of the incident wave, the reflected wave, and the transmitted wave; $E_{0}, A_{0}$, and $C_{s}$ are, respectively, Young's modulus, the cross-sectional area, and the elastic wave speed of the bar; $l_{s}$ and $A_{s}$ are the height and the cross-sectional area of the specimen, respectively; and $t$ is the duration time of elastic wave.

Then, the incident energy $W_{i}$, reflected energy $W_{r}$, and transmitted energy $W_{t}$ can be calculated by

$$
W_{j}=\frac{A_{0} C_{s}}{E_{0}} \int_{0}^{t} \sigma_{j}^{2} d t \quad j=i, r, t
$$

where $\sigma_{i}(t), \sigma_{r}(t)$, and $\sigma_{t}(t)$, respectively, represent the stress of incident wave, reflected wave, and transmitted wave.

In the SHPB test, the incident energy $\left(W_{i}\right)$ caused by the impact between the striker bar and incident bar can be treated as the total energy input to the system, which mainly includes four parts [24]. The energy reflected to the incident bar (reflected energy, $W_{r}$ ), the energy transferred to the transmitted bar (transmitted energy, $W_{t}$ ), the energy absorbed by the specimens (dissipated energy (DE), $W_{s}$ ), and the energy lost at the specimen-incident and specimen-transmitted bar interfaces due to the friction effect, which accounted for a very small proportion, can be ignored. Hence, the energy dissipation $W_{s}$ can be expressed as

$$
W_{s}=W_{i}-W_{r}-W_{t} .
$$

2.4.2. Definition of Strain Rate Sensitivity Coefficient. In order to more clearly and effectively describe the strain rate effect of dynamic compression mechanical properties of saturated yellow sandstone under different low temperatures, the strain rate sensitivity coefficient of peak strength, peak strain, elastic modulus, and energy dissipation were defined as follows [2]:

$$
\left\{\begin{array}{l}
\gamma_{\sigma}=\frac{\sigma_{2}-\sigma_{1}}{\dot{\varepsilon}_{2}-\dot{\varepsilon}_{1}}, \\
\gamma_{\varepsilon}=\frac{\varepsilon_{2}-\varepsilon_{1}}{\dot{\varepsilon}_{2}-\dot{\varepsilon}_{1}}, \\
\gamma_{E}=\frac{E_{2}-E_{1}}{\dot{\varepsilon}_{2}-\dot{\varepsilon}_{1}}, \\
\gamma_{W}=\frac{W_{s 2}-W_{s 1}}{\dot{\varepsilon}_{2}-\dot{\varepsilon}_{1}},
\end{array}\right.
$$

where $\gamma_{\sigma}, \gamma_{\varepsilon}, \gamma_{E}$, and $\gamma_{W}$ are the strain rate sensitivity coefficient of peak strength, peak strain, elastic modulus, and energy dissipation, respectively. $\dot{\varepsilon}_{2}$ and $\dot{\varepsilon}_{1}$ represent the maximum and minimum value of strain rates at each low temperature. $\sigma, \varepsilon, E$, and $W_{s}$ are the corresponding peak stress, peak strain, elastic modulus, and dissipated energy of specimens, respectively. The larger the strain rate sensitivity coefficient is, the more significant the strain rate effect is. 


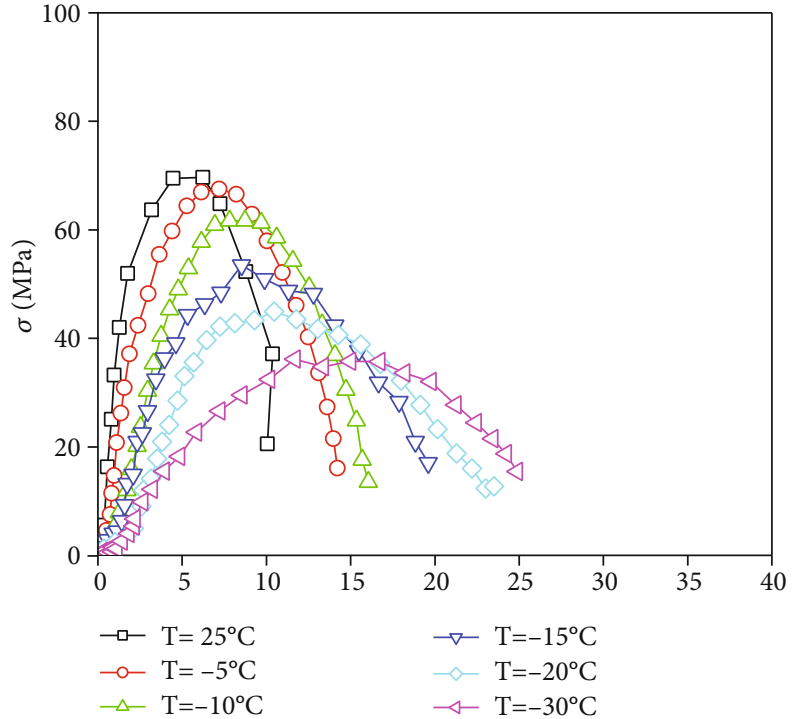

(a) $\dot{\varepsilon}=74.22 \mathrm{~s}^{-1}$

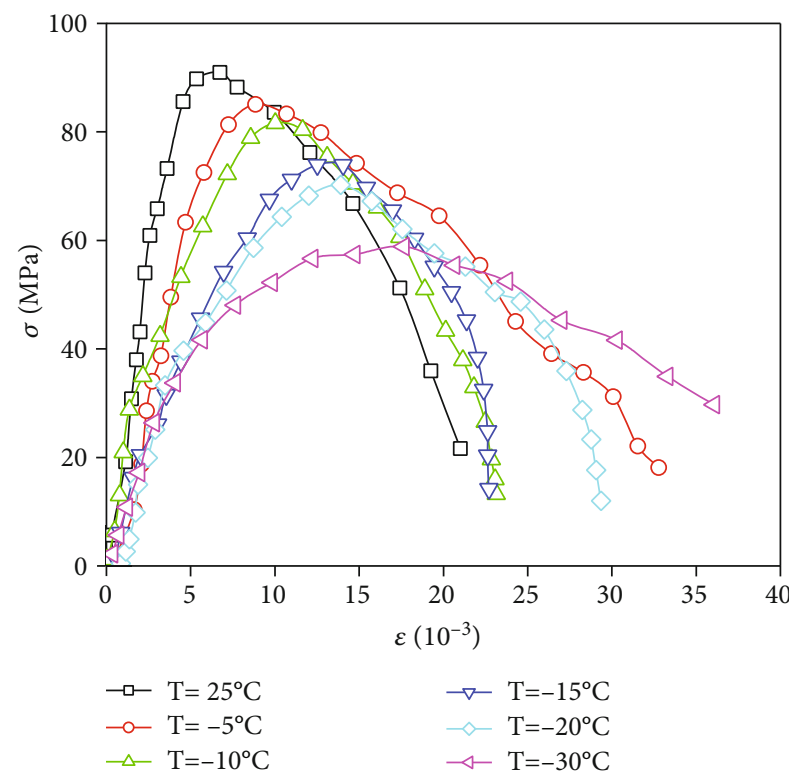

(c) $\dot{\varepsilon}=118.22 \mathrm{~s}^{-1}$

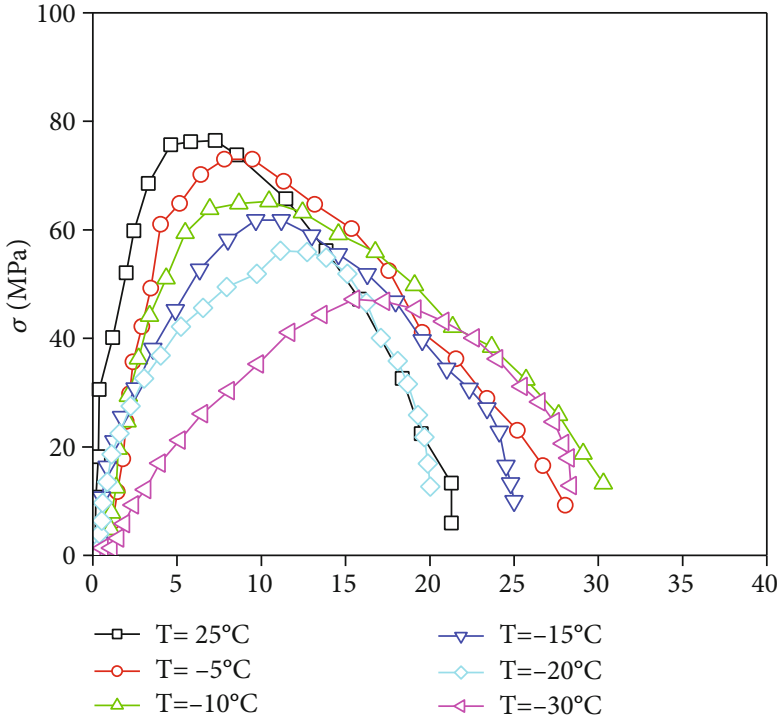

(b) $\dot{\varepsilon}=91.64 \mathrm{~s}^{-1}$

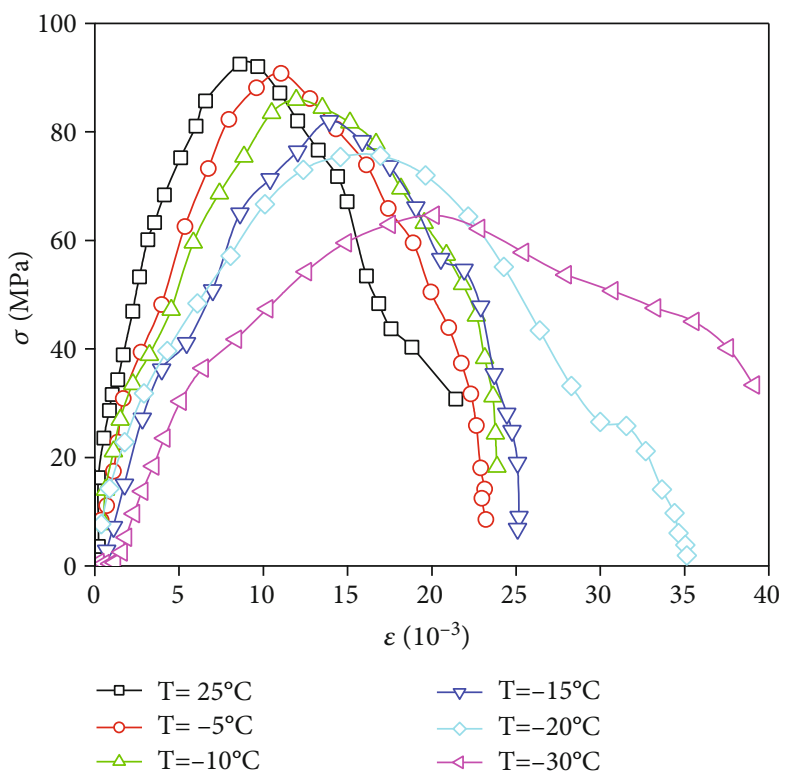

(d) $\dot{\varepsilon}=169.41 \mathrm{~s}^{-1}$

FIgURE 4: The dynamic compression stress-strain curve of saturated yellow sandstone under different temperatures and strain rates.

\section{Experimental Result Analysis}

The dynamic peak stress, dynamic peak strain, dynamic elastic modulus, and brittle/ductile behaviour of specimens under the coupling effect of freeze-thaw temperatures and strain rates could be inferred from the stress-strain curve. Figure 4 presented the typical dynamic compressive stressstrain curves obtained from the SHPB test at four loading levels and six freeze-thaw temperature levels. It can be seen clearly that the strain rates and freeze-thaw temperatures have an extremely important influence on the dynamic compression behavior of rock specimens [29]. The stress-strain curve can be roughly divided into four stages as a whole: nonlinear compression deformation stage with the initial crack closure, elastic deformation stage, crack unsteady propagation stage, and postpeak plastic deformation stage. Although under different strain rates and temperatures, the shape feature of the stress-strain curve is basically similar with their process of nonlinear compression deformation stage relatively short. When the temperature is less than $-20^{\circ} \mathrm{C}$, the slope of the post-peak stress-strain curve decreases significantly. It means that the brittleness of the yellow sandstone specimens weakens whereas their plastic or ductile enhances. In addition, the strength parameters, such as peak stress, peak strain, dynamic elastic modulus, and dissipated energy, of specimens varied as the temperature and strain rates changed. The experimental results of dynamic compressive mechanical behavior for yellow sandstone under different temperatures and strain rates are shown in Table 1. It should be noted that the experiment 
TABLE 1: Experimental result of dynamic compressive mechanical behavior for yellow sandstone under different temperatures and strain rates.

\begin{tabular}{|c|c|c|c|c|c|c|c|}
\hline Number & $\begin{array}{c}\text { Temperature } T \\
\left({ }^{\circ} \mathrm{C}\right)\end{array}$ & $\begin{array}{l}\text { Strain rate } \dot{\varepsilon} \\
\qquad\left(\mathrm{s}^{-1}\right)\end{array}$ & $\begin{array}{l}\text { Average value } \dot{\varepsilon} \\
\qquad\left(\mathrm{s}^{-1}\right)\end{array}$ & $\begin{array}{l}\text { Dynamic compressive strength } \\
(\mathrm{MPa})\end{array}$ & $\begin{array}{l}\text { Peak strain } \\
\qquad\left(10^{-3}\right)\end{array}$ & $\begin{array}{l}\text { Elastic modulus } \\
\quad(\mathrm{GPa})\end{array}$ & $\begin{array}{l}\mathrm{DE} \\
(\mathrm{J})\end{array}$ \\
\hline 1 & 25 & 72.51 & \multirow{6}{*}{74.22} & 70.41 & 5.02 & 34.42 & 30.13 \\
\hline 2 & -5 & 74.57 & & 67.16 & 6.70 & 23.48 & 27.81 \\
\hline 3 & -10 & 76.00 & & 61.83 & 7.84 & 13.46 & 24.77 \\
\hline 4 & -15 & 73.87 & & 53.55 & 8.55 & 9.13 & 23.52 \\
\hline 5 & -20 & 75.02 & & 44.97 & 10.40 & 7.97 & 24.20 \\
\hline 6 & -30 & 73.95 & & 36.39 & 11.72 & 3.68 & 20.19 \\
\hline 1 & 25 & 92.35 & \multirow{6}{*}{91.64} & 76.49 & 6.61 & 35.36 & 48.64 \\
\hline 2 & -5 & 91.58 & & 73.68 & 8.16 & 17.46 & 43.55 \\
\hline 3 & -10 & 91.10 & & 66.20 & 9.14 & 11.91 & 38.93 \\
\hline 4 & -15 & 92.47 & & 62.92 & 10.36 & 5.69 & 34.00 \\
\hline 5 & -20 & 91.93 & & 56.84 & 11.91 & 4.83 & 34.45 \\
\hline 6 & -30 & 90.41 & & 47.49 & 15.50 & 3.08 & 30.69 \\
\hline 1 & 25 & 116.94 & \multirow{6}{*}{118.22} & 90.94 & 6.61 & 23.92 & 72.22 \\
\hline 2 & -5 & 118.27 & & 85.67 & 8.70 & 21.56 & 61.74 \\
\hline 3 & -10 & 119.33 & & 82.46 & 10.26 & 17.71 & 54.54 \\
\hline 4 & -15 & 118.93 & & 74.85 & 12.44 & 9.45 & 49.74 \\
\hline 5 & -20 & 117.86 & & 70.76 & 13.57 & 7.63 & 46.08 \\
\hline 6 & -30 & 117.99 & & 60.23 & 17.30 & 6.42 & 44.74 \\
\hline 1 & 25 & 168.43 & \multirow{6}{*}{169.41} & 92.42 & 8.61 & 23.46 & 85.47 \\
\hline 2 & -5 & 169.35 & & 90.38 & 11.04 & 12.48 & 82.66 \\
\hline 3 & -10 & 170.39 & & 86.30 & 12.09 & 10.33 & 78.87 \\
\hline 4 & -15 & 169.47 & & 82.22 & 14.00 & 8.82 & 66.74 \\
\hline 5 & -20 & 168.98 & & 76.09 & 15.57 & 8.53 & 63.41 \\
\hline 6 & -30 & 169.84 & & 65.02 & 19.57 & 7.70 & 54.23 \\
\hline
\end{tabular}

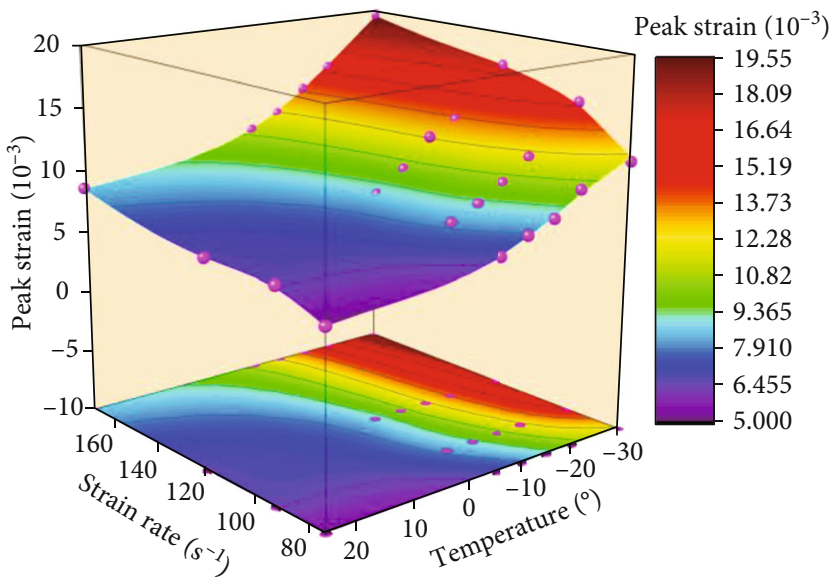

FIGURE 5: Dynamic peak strain under different temperatures and strain rates.

results are the average value of three specimens after removing two groups' data with larger error.

\subsection{Coupling Effect of Strain Rate and Low Temperatures on} Dynamic Peak Strain. Figure 5 shows dynamic peak strain under different temperatures and strain rates. It can be seen clearly that the two variables exist good regularity and the uniform fitting equation of these curves can be expressed as

$$
\varepsilon_{\mathrm{c}}=\left[d_{1}(T) \dot{\varepsilon}+d_{2}(T)\right] \times 10^{-3},
$$

where $d_{1}(T)$ and $d_{2}(T)$ are, respectively, the fitting 
TABLe 2: Fitting coefficient values in Equation (5).

\begin{tabular}{lllllll}
\hline & $25^{\circ} \mathrm{C}$ & $-5^{\circ} \mathrm{C}$ & $-10^{\circ} \mathrm{C}$ & $-15^{\circ} \mathrm{C}$ & $-20^{\circ} \mathrm{C}$ & $-30^{\circ} \mathrm{C}$ \\
\hline$d_{1}$ & 0.033 & 0.042 & 0.042 & 0.055 & 0.052 & 0.074 \\
$d_{2}$ & 2.888 & 3.788 & 4.972 & 5.088 & 6.894 & 7.592 \\
$R^{2}$ & 0.906 & 0.969 & 0.976 & 0.922 & 0.968 & 0.867 \\
\hline
\end{tabular}

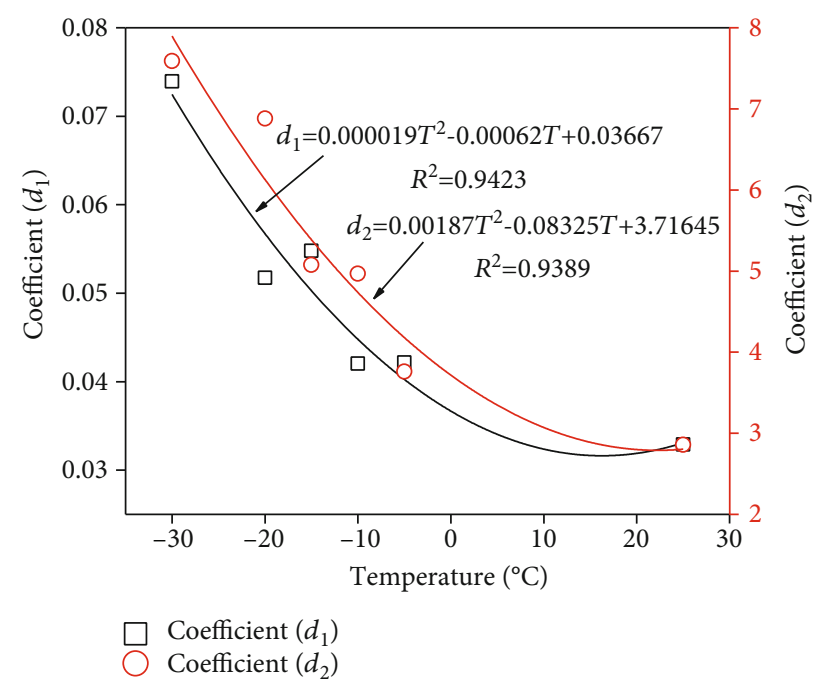

Figure 6: Variation of fitting coefficients with the temperature.

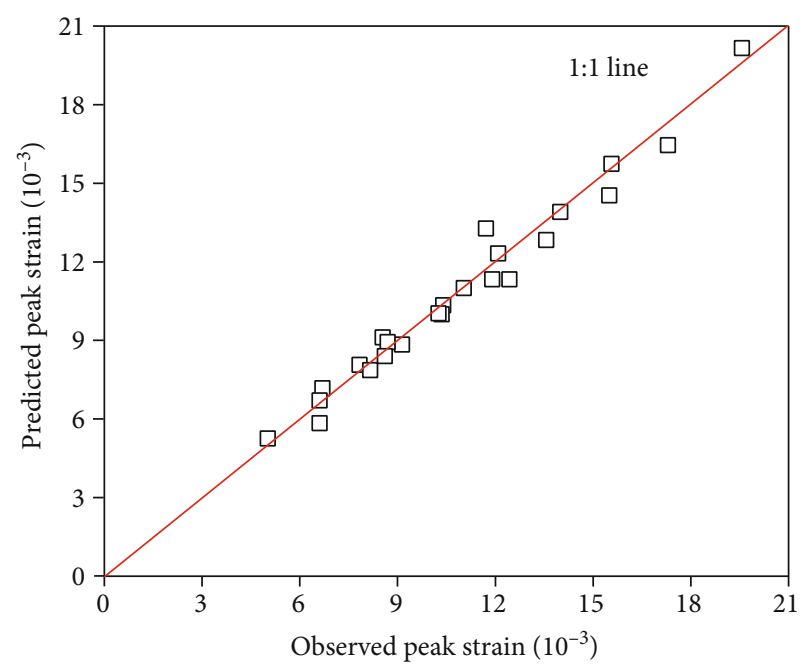

Figure 7: Plots of predicted peak strain against observed peak strain.

coefficients related to temperature, of which the value and correlation coefficient are shown in Table 2.

In order to obtain the specific expression of peak strain under coupling effect of the strain rate and temperature, it is needed to analyze the relationship between the fitting coefficients and temperatures. As shown in Figure 6, the variation of fitting coefficients with the temperature also exists good regularity. The fitting equation is shown in

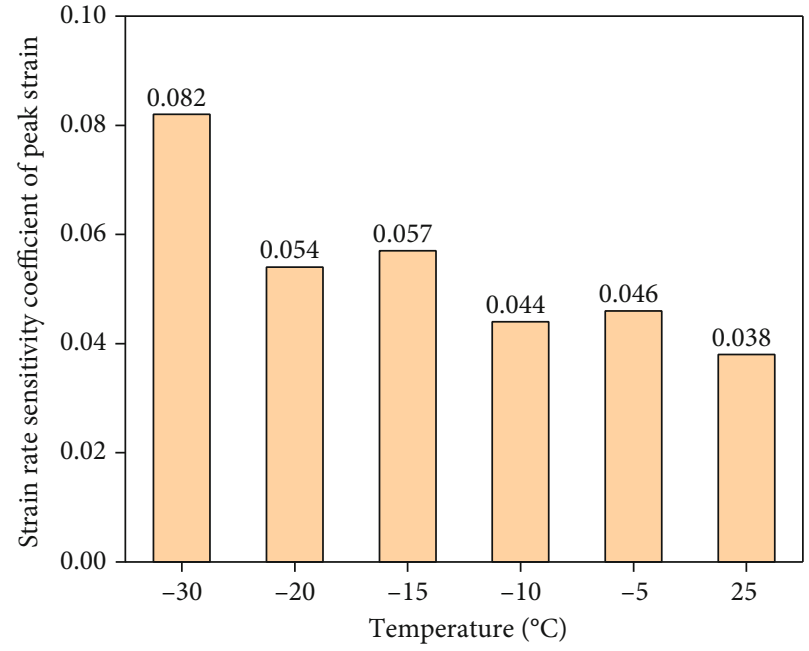

FIGURE 8: Strain rate sensitivity coefficient of peak strain variation with temperature.

$$
\begin{cases}d_{1}=0.000019 T^{2}-0.00062 T+0.03667, & R^{2}=0.9423 \\ d_{2}=0.00187 T^{2}-0.08325 T+3.71645, & R^{2}=0.9389 .\end{cases}
$$

Substituting Equation (6) into Equation (5), the relationship formula of peak strain with strain rate and temperature can be deduced as follows:

$\varepsilon_{c}=\left[\left(0.000019 T^{2}-0.00062 T+0.03667\right) \dot{\varepsilon}+0.00187 T^{2}-0.08325 T+3.71645\right] \times 10^{-3}$

The dynamic peak strain of rock is predicted by Equation (7) and compared with the laboratory observation; as shown in Figure 7, the $1: 1$ inclined line is given in the figure. The distance between the predicted value and the $1: 1$ inclined line represents the error between the predicted value and the observation value [30]. It can be seen from the figure that the predicted value is basically distributed near the 1:1 inclined line; Equation (7) can be used to predict dynamic peak strain at any temperature and impact rate.

Figure 8 shows the strain rate sensitivity coefficient of peak strain variation with temperature. Figure 9 shows the increased ratio of peak strain compared to room temperature under different temperatures and strain rates. It can be seen from Figures 8 and 9 that the enhanced effect of strain rate always exists under low temperature. However, according to the strain rate level $[7,8]$, the increase speed of peak strain at medium strain rate level $\left(74.22 \mathrm{~s}^{-1}\right.$ and $\left.91.64 \mathrm{~s}^{-1}\right)$ is obviously higher than that at high strain rate level $\left(118.22 \mathrm{~s}^{-1}\right.$ and $\left.169.41 \mathrm{~s}^{-1}\right)$ with stain rate increasing. At room temperature, the strain rate sensitivity coefficient of peak strain is 0.038 . With temperature decreasing, the value changes in volatility. From $-5^{\circ} \mathrm{C}$ to $-25^{\circ} \mathrm{C}$, the strain rate sensitivity coefficient increased by $21.05 \%, 15.79 \%$, $50.00 \%$, and $42.11 \%$ compared to room temperature, 


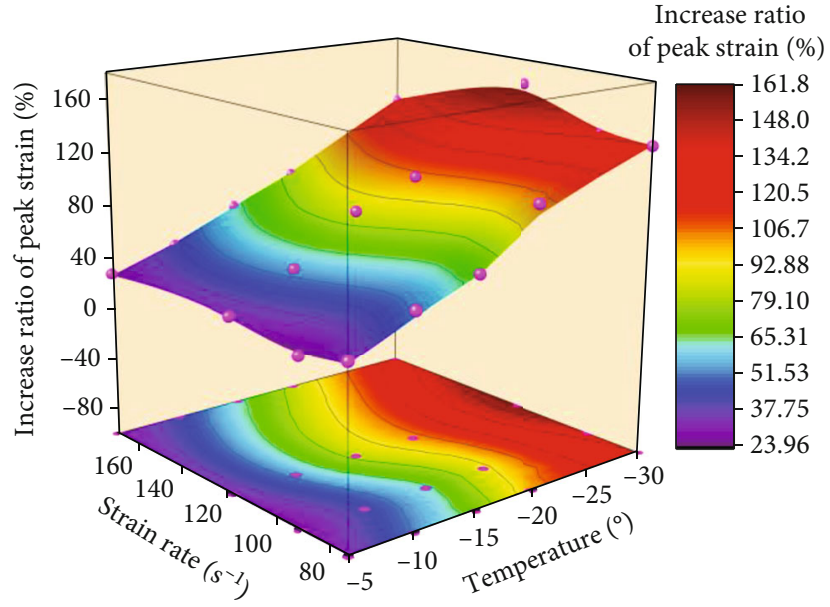

FIGURE 9: Increase ratio of peak strain under different temperatures and strain rates.

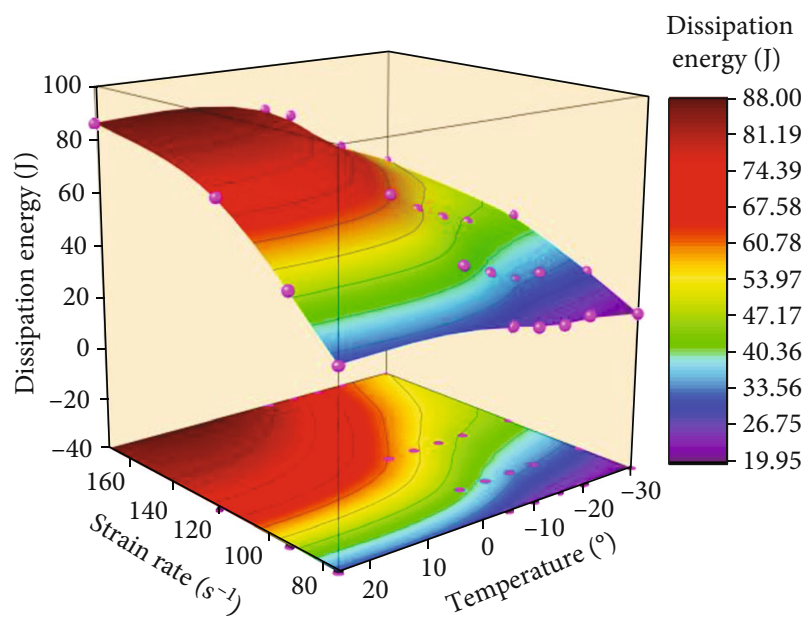

FIgURe 10: Dissipated energy under different temperatures and strain rates.

TABle 3: Fitting coefficient values in Equation (8).

\begin{tabular}{ccccccc}
\hline & $25^{\circ} \mathrm{C}$ & $-5{ }^{\circ} \mathrm{C}$ & $-10{ }^{\circ} \mathrm{C}$ & $-15^{\circ} \mathrm{C}$ & $-20^{\circ} \mathrm{C}$ & $-30^{\circ} \mathrm{C}$ \\
\hline$d_{1}$ & 67.73 & 66.41 & 65.22 & 53.04 & 47.34 & 41.73 \\
$d_{2}$ & 258.00 & 257 & 256.10 & 204.80 & 179.60 & 157.90 \\
$R^{2}$ & 0.956 & 0.996 & 0.999 & 0.997 & 0.999 & 0.972 \\
\hline
\end{tabular}

respectively. When the temperature reach $-30^{\circ} \mathrm{C}$, the strain rate sensitivity coefficient reaches the maximum value of 0.082 increased by $115.79 \%$, indicating that the strain rate effect has a most important influence at this temperature.

In addition, it can be seen from Figure 9 that the increase ratio of peak strain compared to room temperature shows the scattered distribution with the increase of strain rate, indicating that the influence of strain rate on the increase ratio of peak strain is not regular. However, the increase ratio of peak strain gradually increases with decreasing of

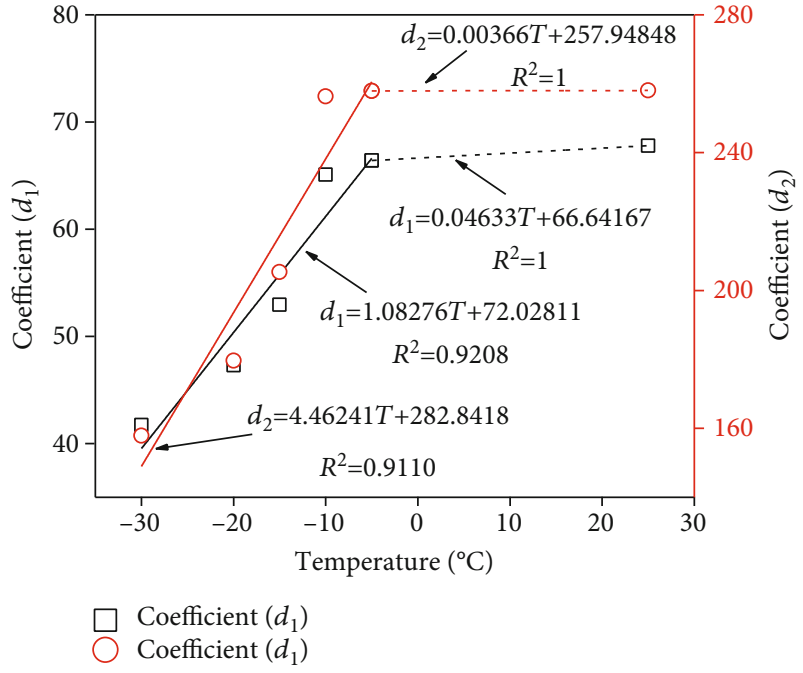

FIGURE 11: Variation of fitting coefficients with the temperature.

temperature and reaches the maximum value when the temperature reaches $-30^{\circ} \mathrm{C}$. It means that the freeze-thaw temperature has a certain enhancement effect on the plastic behavior of yellow sandstone. The lower the freeze-thaw temperature is, the greater the ductility of saturated yellow sandstone is.

3.2. Coupling Effect of Strain Rate and Low Temperatures on Dissipated Energy. Figure 10 shows dissipated energy under different temperatures and strain rates. As can be seen clearly from Figure 10 that the dissipated energy presents obvious strain rate and temperature effects and the uniform fitting equation of these curves is as follows:

$$
W_{L}=d_{1}(T) \ln (\dot{\varepsilon})-d_{2}(T) .
$$

The fitting coefficients $d_{1}(T)$ and $d_{2}(T)$ as well as the correlation coefficient of fitting curves are shown in Table 3 .

For obtaining the certain equation of dissipated energy under coupling influence of strain rate and temperature, the relationship between fitting coefficients and temperature should be firstly determined.

Figure 11 shows the fitting curve of the above variables, indicating a good regularity. The fitting equation can be written as shown in Equation (9).

$$
\begin{aligned}
& d_{1}=\left\{\begin{array}{lll}
1.08276 T+72.02811, & -30 \leq T \leq-5, & R^{2}=0.9208, \\
0.04633 T+66.64167, & -5<T \leq 25, & R^{2}=1,
\end{array}\right. \\
& d_{2}=\left\{\begin{array}{lll}
4.46241 T+282.8418, & -30 \leq T \leq-5, & R^{2}=0.9110, \\
0.00366 T+257.9485, & -5<T \leq 25, & R^{2}=1 .
\end{array}\right.
\end{aligned}
$$

Substituting Equation (9) into Equation (8), the certain expression of dissipated energy under the coupling effect of 


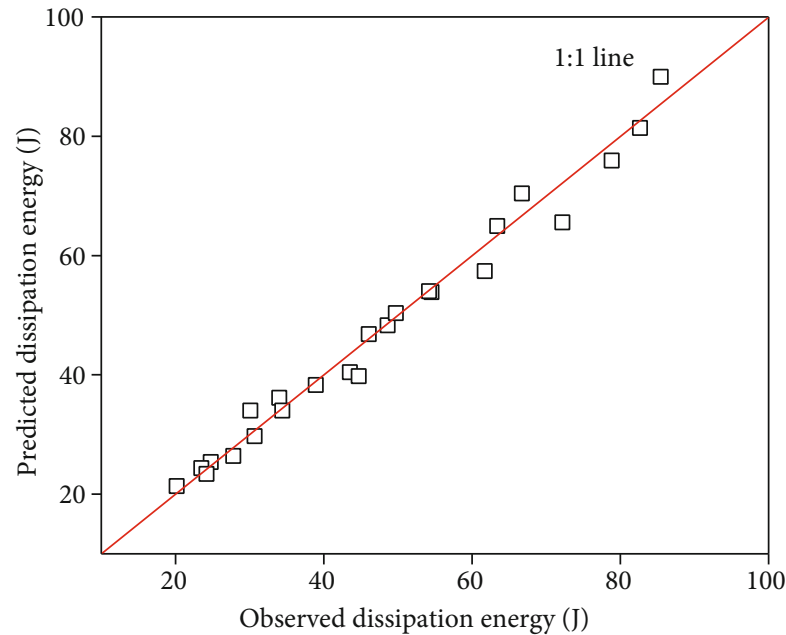

FIGURE 12: Plots of predicted dissipation energy against observed dissipation energy.

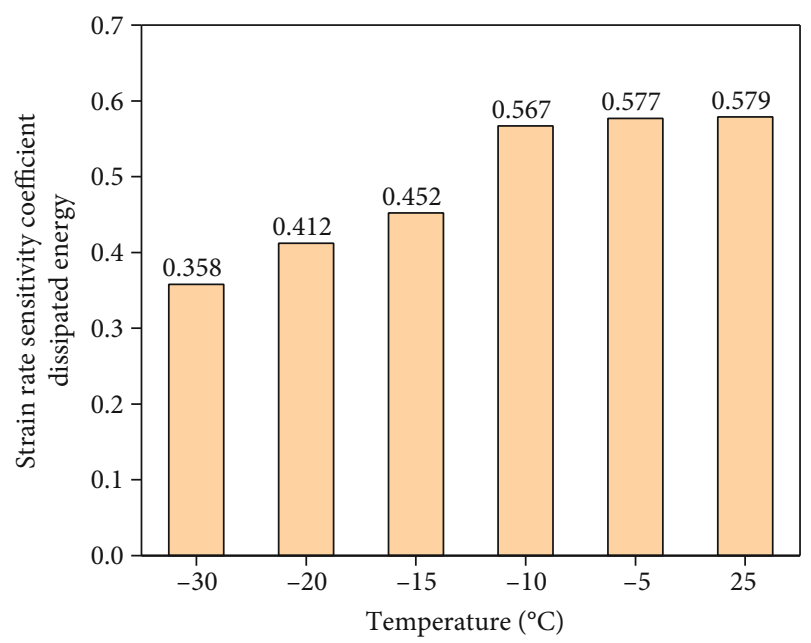

FIGURE 13: Strain rate sensitivity coefficient of dissipated energy variation with temperature.

strain rate and temperature can be obtained as follows:

$W_{L}= \begin{cases}(1.08276 T+72.02811) * \ln (\dot{\varepsilon})-4.46241 T-282.8418, & -30 \leq T \leq-5, \\ (0.04633 T+66.64167) * \ln (\dot{\varepsilon})-0.00366 T-257.9485, & -5<T \leq 25 .\end{cases}$

Equation (10) is used to predict the dissipated energy of rock and compare it with the laboratory observation value. As shown in Figure 12, the 1:1 inclined line is given in the figure. The distance between the predicted value and the 1:1 inclined line represents the error between the predicted value and the observation value. It can be seen from the figure that the predicted value is basically distributed near the 1:1 inclined line; Equation (11) can be used to predict the dissipated energy at any temperature and impact rate.

Figure 13 shows the strain rate sensitivity coefficient of dissipated energy variation with temperature. As can be seen from Figure 13 that the enhancement effect of strain rate on dissipated energy under different temperatures also exists and the increase speed under different temperatures also have an obvious strain rate effect. The increased ratio of dissipated energy at high strain rate level $\left(118.22 \mathrm{~s}^{-1}\right.$ and $\left.169.41 \mathrm{~s}^{-1}\right)$ is lower than that at medium strain rate level $\left(74.22 \mathrm{~s}^{-1}\right.$ and $\left.91.64 \mathrm{~s}^{-1}\right)$, indicating that the strain rate effect gradually weakens with strain rate increasing. At room temperature, the strain rate sensitivity coefficient of dissipated energy is 0.579 . As the temperature goes down, the value gradually nonlinear decreases until it reaches minimum value $(0.358)$ at $-30^{\circ} \mathrm{C}$, showing that the strain rate effect on dissipated energy gradually weakens with the decrease of freeze-thaw temperature.

Figure 14 shows the increase ratio of dissipated energy compared to room temperature under different temperatures and strain rates. It can be seen from Figure 14 that the increased ratio of dissipated energy under different freeze-thaw temperatures shows a change characteristic of decreasing first in the range of $74.22 \mathrm{~s}^{-1} \sim 118.22 \mathrm{~s}^{-1}$ and then increasing in the range of $118.22 \mathrm{~s}^{-1} \sim 169.41 \mathrm{~s}^{-1}$, indicating that the influence of strain rate on the increase ratio of dissipated energy has an obvious interval effect. On the whole, with the increase of freeze-thaw temperature, regardless of individual discrete points, the increase ratio of dissipated energy gradually increases, of which the values are substantially negative. From $-30^{\circ} \mathrm{C}$ to $-5^{\circ} \mathrm{C}$, the increase ratio changes from $-36.9 \%$ to $-10.47 \%$ at strain rate $91.64 \mathrm{~s}^{-1}$ or from $-36.55 \%$ to $-3.29 \%$ at strain rate $118.22 \mathrm{~s}^{-1}$. At $-30^{\circ} \mathrm{C}$, the increase ratio of dissipated energy reaches minimum value, indicating that the energy absorption capacity of rock material has reached weakest.

This maybe mainly because that the freeze-thaw temperature has significantly resulted in the serious inner damage degree of rock material by the frost heaving effect. As a result, the energy absorbed by specimens will be greatly reduced in the process of impact test.

3.3. Coupling Effect of Strain Rate and Low Temperatures on Elastic modulus. The dynamic elastic modulus is an extremely important mechanics index for evaluating the impact deformation resistance of rock materials. In general, there are many various methods to determine elastic modulus, which mainly include secant modulus, tangent modulus, and average modulus. In this paper, we take the slope of approximate straight line at the elastic stage of the stressstrain curve as the elastic modulus of rock material. The methods have already been used in other papers [9].

Figure 15 shows the elastic modulus evolution law under different temperatures and strain rates. Figure 16 shows the strain rate sensitivity coefficient of elastic modulus under different temperatures. As can be seen from Figures 15 and 16, the elastic modulus fluctuates with the strain rate increasing. It means that there is no obvious strain rate effect on the elastic modulus under different temperatures. The experimental phenomenon has already been verified in some experiments [2]. In addition, with the freeze-thaw temperature increasing, the elastic modulus gradually increases, indicating that the lower the freeze-thaw temperature is, the 


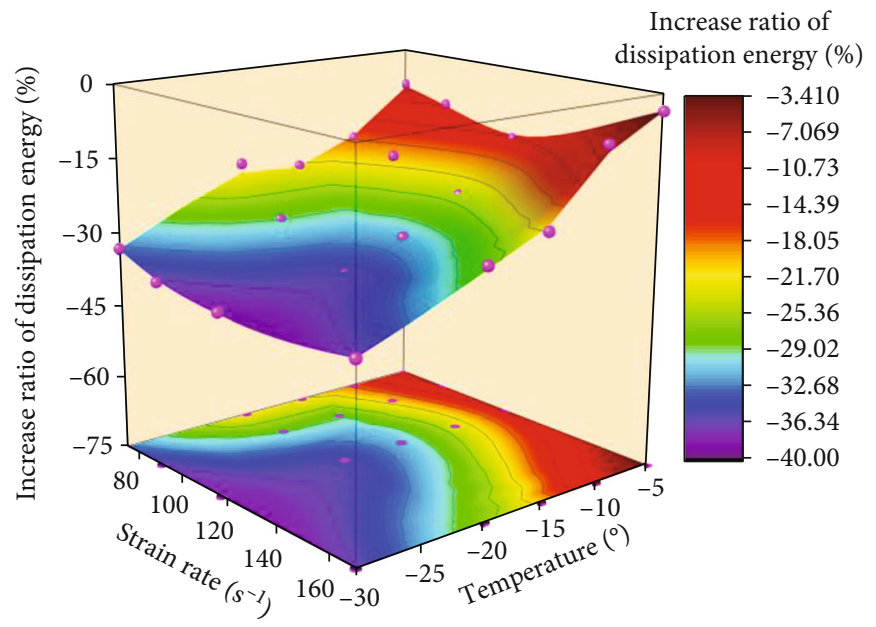

FIGURE 14: Increase ratio of dissipated energy under different temperatures and strain rates.

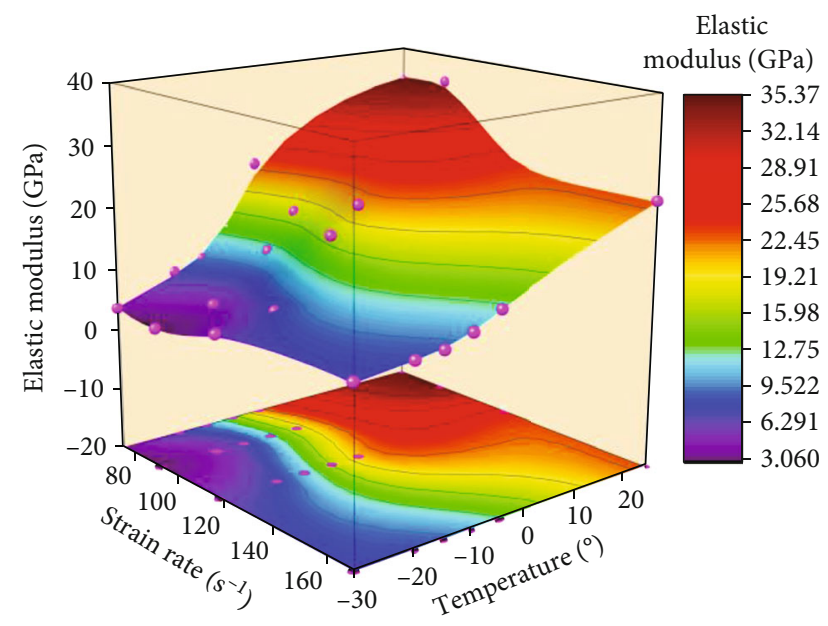

FIGURE 15: Elastic modulus under different temperatures and strain rates.

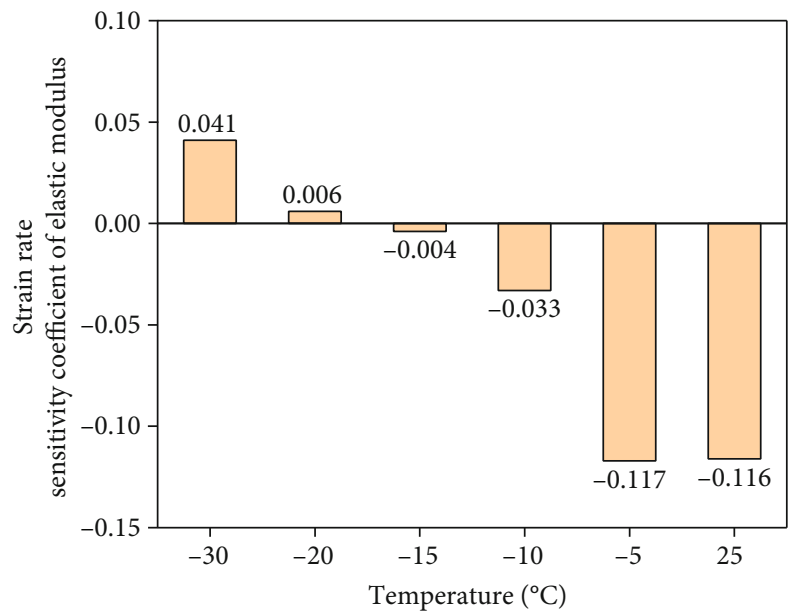

FIGURE 16: Strain rate sensitivity coefficient of elastic modulus variation with temperature. weaker the deformation resistance of yellow sandstone will be.

Figure 17 shows the increase ratio of elastic modulus compared to room temperature. As can be seen from Figure 17, the increased ratio of the elastic modulus presents obvious fluctuation characteristic with strain rate increasing. In addition, the increased ratio of the elastic modulus gradually decreases with the temperature decreasing. From $-5^{\circ} \mathrm{C}$ to $-30^{\circ} \mathrm{C}$, the increase ratio changes from $-31.77 \%$ to $-89.3 \%$ at strain rate $74.22 \mathrm{~s}^{-1}$ or from $-9.8 \%$ to $-73.14 \%$ at strain rate $118.22 \mathrm{~s}^{-1}$. At $-30^{\circ} \mathrm{C}$, the increase ratio of elastic modulus compared to room temperature reaches minimum value, indicating that the ability to resist deformation of rocks has reached weakest under this temperature.

3.4. Coupling Effect of Strain Rates and Low Temperatures on Dynamic Peak Strength. Figure 18 shows dynamic peak stress under different temperatures and strain rates. Figure 19 indicates the strain rate sensitivity coefficient of peak stress variation with temperature. As can be seen from Figures 18 and 19, the enhanced effect of strain rate on peak strength of yellow sandstone under different temperatures also always exists. It has an obvious regularity with temperature and strain rate. On the whole, the increase speed of peak stress at high strain rate level $\left(118.22 \mathrm{~s}^{-1}\right.$ and $169.41 \mathrm{~s}$ $\left.{ }^{1}\right)$ is lower than that at medium strain rate level $\left(74.22 \mathrm{~s}^{-1}\right.$ and $91.62 \mathrm{~s}^{-1}$ ), showing that, at the same temperature, the strain rate effect gradually weakens with the increase of strain rate. In addition, the strain rate sensitivity coefficient of peak stress is always positive and dramatically increases with the temperature decreasing from $25^{\circ} \mathrm{C}$ to $-20^{\circ} \mathrm{C}$, indicating that the strain rate effect on peak strength gradually strengthened with the decrease of freeze-thaw temperature. Otherwise, the strain rate sensitivity coefficient of 0.30 at $-30^{\circ} \mathrm{C}$ is slightly lower than that of 0.33 at $-20^{\circ} \mathrm{C}$, but higher than that under other temperatures. It may be because that, at $-30^{\circ} \mathrm{C}$, the surface of specimens appeared an obvious particle spalling phenomenon, which increased the frost heave damage degree and destroyed the integrity of rock material 


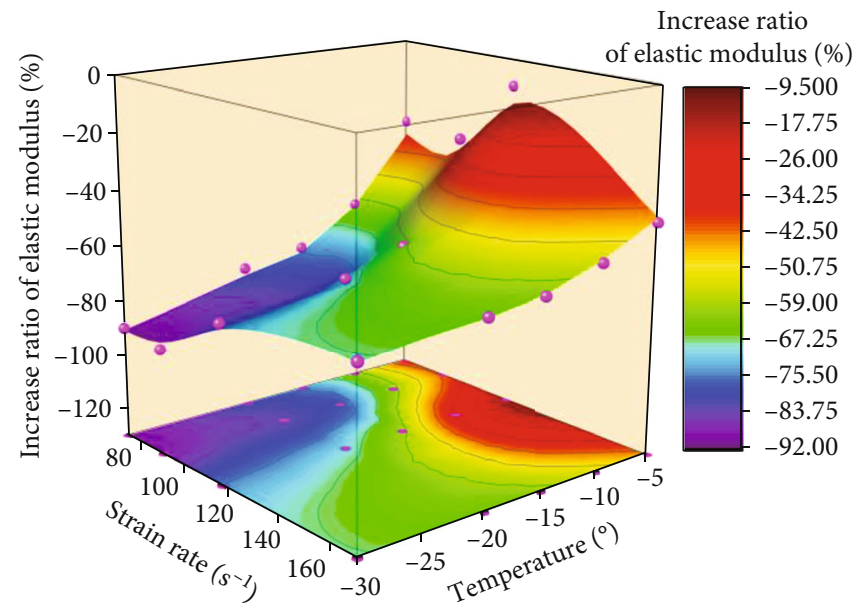

FIGURE 17: Increased ratio of the elastic modulus under different temperatures and strain rates.

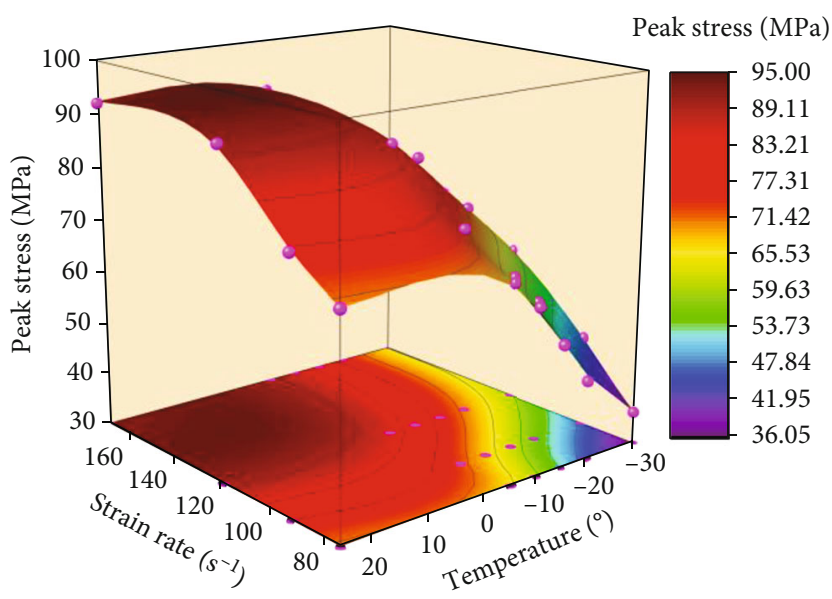

FIGURE 18: Dynamic peak stress under different temperatures and strain rates.

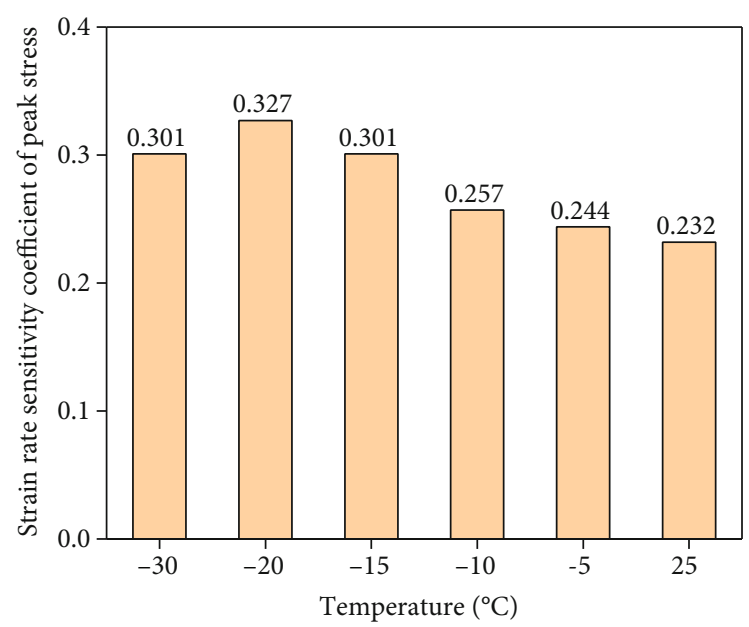

FIGURE 19: Strain rate sensitivity coefficient of peak stress variation with temperature.

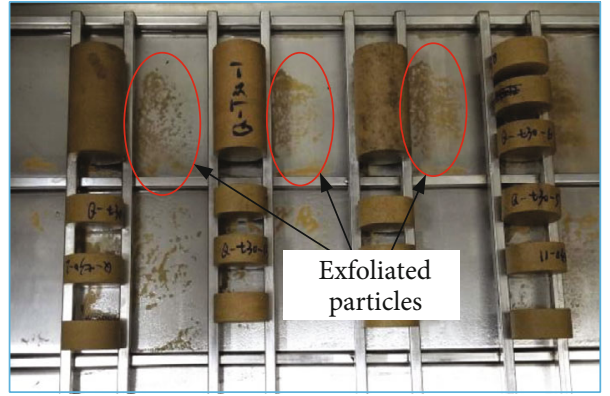

FIgURe 20: Particle spalling phenomenon at $-30^{\circ} \mathrm{C}$ under different size specimens.

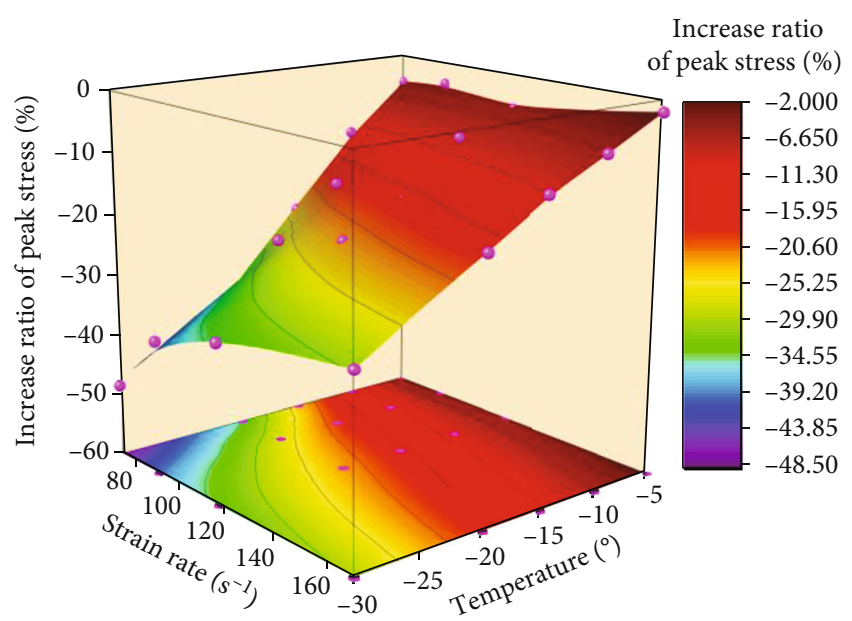

FIgURE 21: Increased ratio of peak stress compared to the room temperature under different temperatures and strain rates.

so that caused errors in the test results. The particle spalling phenomenon is shown in Figure 20.

Figure 21 shows the increase ratio of peak stress compared to the room temperature under different temperatures and strain rates. It can be seen from Figure 21 that, on the whole, the increase ratio of peak stress has an obvious 
TABLE 4: Fractal characteristic parameters of fractured sandstone.

\begin{tabular}{|c|c|c|c|c|c|c|c|c|c|c|c|}
\hline$T\left({ }^{\circ} \mathrm{C}\right)$ & $\dot{\varepsilon}\left(\mathrm{s}^{-1}\right)$ & \multicolumn{8}{|c|}{$M_{\mathrm{R}} / M$} & $d$ & $D$ \\
\hline \multirow{4}{*}{-5} & 74.22 & 0.13 & 0.11 & 0.09 & 0.06 & 0.04 & 0.03 & 0.02 & 0.02 & 0.99 & 2.01 \\
\hline & 91.64 & 0.37 & 0.37 & 0.29 & 0.21 & 0.11 & 0.08 & 0.06 & 0.04 & 0.91 & 2.09 \\
\hline & 118.22 & 0.60 & 0.54 & 0.48 & 0.38 & 0.30 & 0.24 & 0.19 & 0.13 & 0.58 & 2.29 \\
\hline & 169.41 & 0.74 & 0.65 & 0.59 & 0.48 & 0.37 & 0.30 & 0.25 & 0.16 & 0.56 & 2.44 \\
\hline \multirow{4}{*}{-10} & 74.22 & 0.16 & 0.15 & 0.13 & 0.09 & 0.06 & 0.04 & 0.03 & 0.02 & 0.92 & 2.08 \\
\hline & 91.64 & 0.46 & 0.41 & 0.33 & 0.27 & 0.18 & 0.13 & 0.10 & 0.07 & 0.72 & 2.28 \\
\hline & 118.22 & 0.61 & 0.60 & 0.54 & 0.48 & 0.38 & 0.28 & 0.23 & 0.15 & 0.54 & 2.46 \\
\hline & 169.41 & 0.89 & 0.84 & 0.82 & 0.71 & 0.60 & 0.48 & 0.39 & 0.26 & 0.47 & 2.53 \\
\hline \multirow{4}{*}{-15} & 74.22 & 0.24 & 0.23 & 0.16 & 0.11 & 0.06 & 0.05 & 0.04 & 0.02 & 0.90 & 2.10 \\
\hline & 91.64 & 0.54 & 0.53 & 0.48 & 0.37 & 0.26 & 0.19 & 0.15 & 0.10 & 0.67 & 2.33 \\
\hline & 118.22 & 0.69 & 0.64 & 0.55 & 0.45 & 0.36 & 0.29 & 0.25 & 0.17 & 0.52 & 2.48 \\
\hline & 169.41 & 0.92 & 0.92 & 0.90 & 0.85 & 0.69 & 0.56 & 0.51 & 0.34 & 0.38 & 2.62 \\
\hline \multirow{4}{*}{-20} & 74.22 & 0.35 & 0.30 & 0.28 & 0.16 & 0.10 & 0.08 & 0.06 & 0.04 & 0.82 & 2.18 \\
\hline & 91.64 & 0.61 & 0.54 & 0.52 & 0.42 & 0.34 & 0.27 & 0.22 & 0.14 & 0.60 & 2.40 \\
\hline & 118.22 & 0.68 & 0.68 & 0.67 & 0.58 & 0.47 & 0.38 & 0.32 & 0.21 & 0.48 & 2.52 \\
\hline & 169.41 & 0.96 & 0.96 & 0.93 & 0.89 & 0.77 & 0.64 & 0.55 & 0.37 & 0.36 & 2.64 \\
\hline \multirow{4}{*}{-30} & 74.22 & 0.44 & 0.44 & 0.35 & 0.29 & 0.21 & 0.16 & 0.13 & 0.08 & 0.65 & 2.35 \\
\hline & 91.64 & 0.71 & 0.65 & 0.59 & 0.47 & 0.37 & 0.29 & 0.25 & 0.16 & 0.57 & 2.43 \\
\hline & 118.22 & 0.72 & 0.70 & 0.68 & 0.57 & 0.45 & 0.36 & 0.30 & 0.21 & 0.45 & 2.55 \\
\hline & 169.41 & 0.97 & 0.97 & 0.96 & 0.91 & 0.83 & 0.71 & 0.61 & 0.44 & 0.30 & 2.70 \\
\hline
\end{tabular}

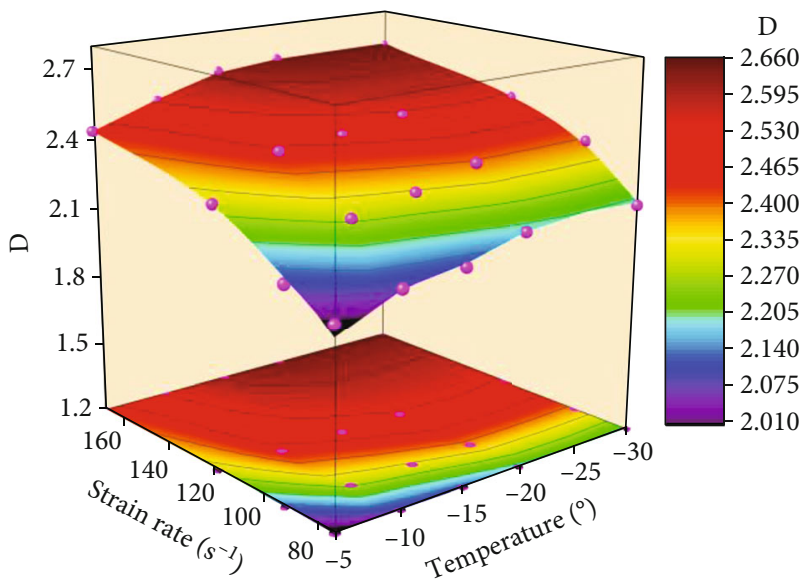

FIGURE 22: Variation of fractal dimension, $D$, with strain rate and freeze-thaw temperature.

correlation with the temperature and strain rate. As the strain rate increases, the increase ratio gradually nonlinear increases and the phenomenon is more significant with the temperature decreasing. When the temperature reaches $-30^{\circ} \mathrm{C}$, the phenomenon is most pronounced. In addition, the increase ratio of peak stress gradually decreases with decreasing of temperature and reaches the minimum value of $-48.32 \%$ for $74.22 \mathrm{~s}^{-1}, 37.92 \%$ for $91.64 \mathrm{~s}^{-1}, 33.76 \%$ for $118.22 \mathrm{~s}^{-1}$ and $29.65 \%$ for $169.41 \mathrm{~s}^{-1}$ when the temperature reaches $-30^{\circ} \mathrm{C}$, indicating that the freeze-thaw temperature has a serious weakening effect on rock strength, which can reduce the bearing capacity of rocks.

\section{Coupling Effect of Strain Rate and Low Temperature on Fractal Characteristics}

4.1. Fractal Dimension Calculation Methods. In order to quantitatively study the effect of strain rate on the fracture degree of specimens at different freeze-thaw temperatures, the mass-equivalent dimension method as an effective fractal dimension calculation method is used to explore the fractal characteristics of the fracture specimens after impact test in this literature. The specific calculation formula is as follows [27]:

$$
\begin{gathered}
D=3-d, \\
d=\frac{\lg \left(M_{R} / M\right)}{\lg R},
\end{gathered}
$$

where $D$ is the fractal dimension of fracture specimens. The larger parameter $D$ is, the higher the fracture degree of specimen is. $d$ is the slope of the fitting line in the logarithmic coordinates $\left(\lg \left(M_{R} / M\right)-\lg R\right) . M_{R}$ is the cumulative mass of fragments smaller than particle size $R$ for each specimen. $M$ is the total mass of fragment.

After the impact test, the fragment of specimen firstly be collected, and then, a classifying screen with eight layers is used to divide the particle size range of fragments into 50- 
$15 \mathrm{~mm}, \quad 15-13 \mathrm{~mm}, \quad 13-11 \mathrm{~mm}, 11-8.5 \mathrm{~mm}, 8.5-6 \mathrm{~mm}, 6-$ $4 \mathrm{~mm}, 4-2.5 \mathrm{~mm}, 2.5-1 \mathrm{~mm}$, and $1-0 \mathrm{~mm}$. The maximum particle size is $50 \mathrm{~mm}$. The fragment mass in different ranges of particle size will be measured by electronic scales and the parameters $M, M_{R}$, and $M_{R} / M$ will also be calculated. Next, parameter $d$ can be obtained by taking the slope of fitting line in the logarithmic coordinates $\left(\lg \left(M_{R} / M\right)-\lg R\right)$. Finally, substituting parameter $d$ into Equation (11), the fractal dimension of fragments $(D)$ can be easily calculated at different temperatures and strain rates. The fractal characteristic parameters of fractured sandstone are shown in Table 4.

4.2. Variation of Fractal Dimension under Strain Rate and Freeze-Thaw Temperature. Figure 22 shows the variation of fractal dimension with strain rate and freeze-thaw temperature.

It can be seen from Figure 22 that the strain rate effect on the fractal dimension under different freeze-thaw temperatures always exists. The fractal dimension presents a positive correlation with strain rate. When the strain rate increases from $74.22 \mathrm{~s}^{-1}$ to $169.41 \mathrm{~s}^{-1}$, the fractal dimension of fragments increases from 2.01 to 2.44 , up $21.39 \%$ for $-5^{\circ} \mathrm{C}$; from 2.08 to 2.53 , up $21.63 \%$ for $-10^{\circ} \mathrm{C}$; from 2.10 to 2.62 , up $24.76 \%$ for $-15^{\circ} \mathrm{C}$; from 2.18 to 2.64 , up $21.10 \%$ for $-20^{\circ} \mathrm{C}$; and from 2.38 to 2.70 , up $14.89 \%$ for $-30^{\circ} \mathrm{C}$. In addition, on the whole, the increased speed of fractal dimension gradually decreases with strain rate increasing, showing that high strain rate is more likely to increase the fracture degree of rock material, but the strain rate effect weakened at high strain rate. In addition, the fractal dimension also gradually increases with freeze-thaw temperature decreasing, indicating that the low freeze-thaw temperature environment has a positive function for increasing the damage and fracture degree of specimens for the saturated specimen. When the temperature reaches $-30^{\circ} \mathrm{C}$ and the strain rate reaches $169.41 \mathrm{~s}^{-1}$, the fractal dimension reaches the maximum value, indicating that the fracture degree of specimens at this state is the most serious.

\section{Conclusion}

In this paper, the coupling effect of strain rate and freezethaw temperature on dynamic compressive mechanical and fracture behavior of saturated yellow sandstone are studied by SHPB test. Moreover, the relationship formula of the peak strain and dissipated energy with the strain rate and freezethaw temperature is also established. Finally, the fractal dimension method is introduced to analyze the fractal characteristics of fractured specimens subjected to different strain rates and temperatures after impact test. The main conclusions summed up are as follows:

(1) The enhanced effects of strain rates on the peak strain, peak strength, and dissipated energy of saturated yellow sandstone under different freeze-thaw temperatures still exist. With the increase of strain rate, the increase speed of above parameters at medium strain rate level $\left(74.22 \mathrm{~s}^{-1}\right.$ and $\left.91.64 \mathrm{~s}^{-1}\right)$ was obviously higher than that at high strain rate level $\left(118.22 \mathrm{~s}^{-1}\right.$ and $\left.169.41 \mathrm{~s}^{-1}\right)$, indicating that the strain rate effect gradually weakens at high strain. The elastic modulus change has nothing to do with the strain rate

(2) At the same strain rate, the parameters of peak strength, elastic modulus, and dissipated energy gradually decrease with the decrease of freeze-thaw temperature whereas peak strain opposite

(3) At room temperature, the strain rate effect on peak strain and peak strength is weakest, while at $-20^{\circ} \mathrm{C} \sim-30^{\circ} \mathrm{C}$, it is most significant. When the temperature is higher than $-10^{\circ} \mathrm{C}$, the increased speed of strain rate sensitivity coefficient has no obvious change, while the increase rapidly with temperature decreasing when it is below $-10^{\circ} \mathrm{C}$. In addition, the strain rate effect on dissipated energy is significant at room temperature, while weakest at $-30^{\circ} \mathrm{C}$

(4) The strain rate and freeze-thaw temperature have a significantly important effect on the fractal dimension of fractured specimens. With the strain rate increasing, the fractal dimension gradually increases. However, the increased speed gradually decreases, showing that high strain rate is more likely to increase the fracture degree of rock material, but the strain rate effect weakened at high strain rate. In addition, the fractal dimension also gradually increases with freeze-thaw temperature decreasing, indicating that the freeze-thaw temperature environment with low temperature has a positive function for increasing the damage and fracture degree of specimens for saturated specimen

\section{Data Availability}

Most of the data generated or analyzed during this study are included in this manuscript, and all of the data are available from the corresponding author on reasonable request.

\section{Conflicts of Interest}

The authors declare that they have no conflicts of interest.

\section{Acknowledgments}

This work was financially supported by the National Natural Science Foundation of China (52074240, 51974295) and the Research Project of “333 Project” in Jiangsu Province of China (BRA2019236).

\section{References}

[1] D. Asprone, E. Cadoni, F. Iucolano, and A. Prota, "Analysis of the strain-rate behavior of a basalt fiber reinforced natural hydraulic mortar," Cement \& Concrete Composites, vol. 53, pp. 52-58, 2014.

[2] S. Liu and J. Xu, "Effect of strain rate on the dynamic compressive mechanical behaviors of rock material subjected to high 
temperatures," Mechanics of Materials, vol. 82, pp. 28-38, 2015.

[3] M. Li, X. Mao, L. Cao, H. Pu, R. Mao, and A. Lu, "Effects of thermal treatment on the dynamic mechanical properties of coal measures sandstone," Rock Mechanics and Rock Engineering, vol. 49, no. 9, pp. 3525-3539, 2016.

[4] Y. Chen, G. Lin, R. Mao, M. Li, X. Mao, and K. Zhang, "Strain rate effect on the mechanical properties and fracture surface roughness of sandstone subjected to dynamic direct tension," IEEE Access, vol. 8, pp. 107977-107992, 2020.

[5] M. Li, L. Gang, and X. Mao, "Dynamic fracture and energy consumption characteristics of coal-series sandstone after heat treatment," Thermal Science, vol. 23, Suppl. 3, pp. 967-974, 2019.

[6] S. J. Green, J. D. Leasia, R. D. Perkins, and A. H. Jones, "Triaxial stress behavior of Solenhofen limestone and westerly granite at high strain rates," Journal of Geophysical Research, vol. 77 , no. 20, pp. 3711-3724, 1972.

[7] T. L. Blanton, "Effect of strain rates from $10^{-2}$ to $10 \mathrm{sec}^{-1}$ in triaxial compression tests on three rocks," International Journal of Rock Mechanics \& Mining Sciences \& Geomechanics Abstracts, vol. 18, no. 1, pp. 47-62, 1981.

[8] Q. B. Zhang and J. Zhao, "A review of dynamic experimental techniques and mechanical behaviour of rock materials," Rock Mechanics and Rock Engineering, vol. 47, no. 4, pp. 1411-1478, 2014.

[9] M. Li, X. Mao, L. Cao, H. Pu, and A. Lu, "Influence of heating rate on the dynamic mechanical performance of coal measure rocks," International Journal of Geomechanics, vol. 17, no. 8, article 04017020, 2017.

[10] U. S. Lindholm and L. M. Yeakley, "High strain-rate testing: tension and compression," Experimental Mechanics, vol. 8, no. 1, pp. 1-9, 1968.

[11] W. F. Brace and A. H. Jones, "Comparison of uniaxial deformation in shock and static loading of three rocks," Journal of Geophysical Research, vol. 76, no. 20, pp. 4913-4921, 1971.

[12] J. R. Klepaczko, "Behavior of rock-like materials at high strain rates in compression," International Journal of Plasticity, vol. 6, no. 4, pp. 415-432, 1990.

[13] Q. M. Li and H. Meng, "About the dynamic strength enhancement of concrete-like materials in a split Hopkinson pressure bar test," International Journal of Solids and Structures, vol. 40, no. 2, pp. 343-360, 2003.

[14] S. G. Grantham, C. R. Siviour, W. G. Proud, and J. E. Field, "High-strain rate Brazilian testing of an explosive simulant using speckle metrology," Measurement Science \& Technology, vol. 15, no. 9, pp. 1867-1870, 2004.

[15] X. Liu, R. Zhang, and J. Liu, "Dynamic test study of coal rock under different strain rates," Journal of China Coal Society, vol. 37, no. 9, pp. 1528-1534, 2012.

[16] Y. Li, D. Huang, and X. Li, "Strain rate dependency of coarse crystal marble under uniaxial compression: strength, deformation and strain energy," Rock Mechanics and Rock Engineering, vol. 47, no. 4, pp. 1153-1164, 2014.

[17] T. Saksala, "On the strain rate sensitivity of coarse-grained rock: a mesoscopic numerical study," Rock Mechanics and Rock Engineering, vol. 52, no. 9, pp. 3229-3240, 2019.

[18] R. Altindag, I. S. Alyildiz, and T. Onargan, "Mechanical property degradation of ignimbrite subjected to recurrent freezethaw cycles," International Journal of Rock Mechanics and Mining Sciences, vol. 41, no. 6, pp. 1023-1028, 2004.
[19] H. Yavuz, "Effect of freeze-thaw and thermal shock weathering on the physical and mechanical properties of an andesite stone," Bulletin of Engineering Geology and the Environment, vol. 70, no. 2, pp. 187-192, 2011.

[20] X. Tan, W. Chen, J. Yang, and J. Cao, "Laboratory investigations on the mechanical properties degradation of granite under freeze-thaw cycles," Cold Regions Science and Technology, vol. 68, no. 3, pp. 130-138, 2011.

[21] G. Charrier, K. Charra-Vaskou, J. Kasuga, H. Cochard, S. Mayr, and T. Ameglio, "Freeze-thaw stress: effects of temperature on hydraulic conductivity and ultrasonic activity in ten woody angiosperms," Plant Physiology, vol. 164, no. 2, pp. 992-998, 2014.

[22] L. Wen, L. I. Xibing, W. U. Qiuhong, L. Weng, and S. U. Wei, "Dynamic strength of granite porphyry under freezingthawing cycles," Chinese Journal of Rock Mechanics and Engineering, vol. 34, no. 7, pp. 1297-1306, 2015.

[23] J. Eslami, C. Walbert, A. Beaucour, A. Bourges, and A. Noumowe, "Influence of physical and mechanical properties on the durability of limestone subjected to freeze-thaw cycles," Construction and Building Materials, vol. 162, pp. 420-429, 2018.

[24] Q. Ma, D. Ma, and Z. Yao, "Influence of freeze-thaw cycles on dynamic compressive strength and energy distribution of soft rock specimen," Cold Regions Science and Technology, vol. 153, pp. 10-17, 2018.

[25] G. H. Zheng, J. Y. Xu, P. Wang, X. Y. Fang, and M. Wen, "Physical characteristics and degradation model of stratified sandstone under freeze-thaw cycling," Yantu Lixue/Rock and Soil Mechanics, vol. 40, no. 2, pp. 632-641, 2019.

[26] M. Grenon, J. Hadjigeorgiou, and Q. Liu, “Quantifying in-situ rock block size and resulting fragment size distributions due to blasting," Fragblast, vol. 2, no. 2, pp. 205-218, 1998.

[27] D. J. Li, X. N. Jia, J. L. Miao, M. C. He, and D. D. Li, “Analysis of fractal characteristics of fragments from rock burst test of granite," Chinese Journal of Rock Mechanics and Engineering, vol. 29, no. 1, pp. 3280-3289, 2010.

[28] A. H. Bakhshandeh and H. R. Malekinezhad, An Empirical Model to Predict Particle Size Reduction Caused by Blasting in the Conglomerate Rock-Mass at Gotvand Olya Dam, Energy Engineering and Management, 2012.

[29] L. Chen, X. Mao, S. Yang, C. An, and P. Wu, "Experimental investigation on dynamic fracture mechanism and energy evolution of saturated yellow sandstone under different freezethaw temperatures," Advances in Civil Engineering, vol. 2019, Article ID 2375276, 16 pages, 2019.

[30] L. Chen, P. Wu, Y. Chen, and W. Zhang, "Experimental study on physical-mechanical properties and fracture behaviors of saturated yellow sandstone considering coupling effect of freeze-thaw and specimen inclination," Sustainability, vol. 12, no. 3, article 1029, 2020. 